\title{
Detection and Antimicrobial Susceptibility Pattern of Extended Spectrum Beta Lactamases (ESBLs) Producing Gram Negative Bacteria from Different Clinical Samples
}

\author{
Biswas $\mathrm{SM}^{1}$, Ara $\mathrm{N}^{2}$, Huda $\mathrm{N}^{3}$, Andalib $\mathrm{S}^{4}$, Rahman $\mathrm{MH}^{5}$, Mia MRA ${ }^{6}$
}

\begin{abstract}
Introduction: Extended spectrum $\beta$-lactamases (ESBLs) are enzymes that mediate resistance to extended-spectrum (third generation) cephalosporins (e.g., ceftazidime, cefotaxime, and ceftriaxone) and monobactams (e.g., aztreonam) but do not affect cephamycins (e.g., cefoxitin and cefotetan) or carbapenems (e.g., meropenem or imipenem). Though the no. of ESBLs producing organism has been increasing day by day, the detection methods and treatment option for them are extremely limited. Aims \& Objective: The present study was undertaken to investigate the rate of ESBLs production and their antibiotic susceptibility pattern. Materials \& Method: A total 110 Gram negative isolates from various clinical samples from a tertiary care hospital were studied and ESBLs production was detected by double disc synergy test. Antibiotic susceptibility test was done for commonly used antibiotics. Results: Among the total isolates $66.36 \%$ (73) were ESBLs producer, and the rate of ESBLs positivity was $80.32 \%$ for E.coli (49 out of 61), 25\% for Pseudomonas spp (6 out of 24), 71.42\% for Klebsiella spp (10 out of 14), 80\% for Enterobacter spp (4 out of 5), 100\% for Acinetobacter spp (4 out of 4) and 0\% for Proteus spp (0 out of 2). ESBLs producing organisms were resistant to most of the antibiotics but $100 \%$ were sensitive to imepenem. Conclusion: Screening for ESBLs production needs to be carried out routinely in every clinical diagnostic laboratory to guide clinicians in proper selection of antibiotics.
\end{abstract}

Key words

ESBLs, multidrug resistance, double disc synergy test

\section{Introduction}

Enterobacteriaceae producing extended spectrum $\beta$-lactamases (ESBLs) cause inactivation of $\beta$-lactam antibiotics especially the newer third generation cephalosporins. ESBLs producing enterobacteriaceae are also frequently resistant to other groups of commonly used non- $\beta$-lactam antibiotics such as fluroquinolones. ${ }^{1}$ Majority of ESBLs producing strains are Klebsiella pneumoniae,
Klebsiella oxytoca and E.coli. Other organisms reported to harbour ESBLs include Enterobacter spp, Salmonella spp, Morganella morganii, Proteus mirabilis, Serratia marcescens and Pseudomonas aeruginosa. $^{2}$

ESBLs enzymes are mediated by plasmids and are the products of point mutations at the active site of Temoniera (TEM), Sulfhydryl variable (SHV) and Oxacillinase (OXA) enzymes. In addition these plasmids also carry resistance to 


\section{ORIGINAL ARTICLE}

several other antimicrobial agents, an important limitation in the design of treatment alternatives. ${ }^{3}$ It is generally thought that patients suffering from infections caused by an ESBLs-producing organism are at an increased risk of treatment failure with an expanded-spectrum $\beta$-lactam antimicrobials as well as other commonly used antimicrobial agents.

The prevalence of ESBLs among clinical isolates varies from country to country and from institution to institution. ${ }^{4}$ In the United States the occurrences of ESBLs in enterobacteriaceae range from 0 to $25 \%$. In India, the prevalence rate varies in different institutions from 28 to $84 \% .^{5}$ Elsewhere in Asia the percentage of ESBLs production in E.coli and K. pneumoniae varies from $4.8 \%$ in Korea to $8.5 \%$ in Taiwan and up to $12 \%$ in Hong kong. ${ }^{6,7,8}$ A comprehensive study of 2840 isolates collected between April and October 2002 from both hospital and community specimens in Pakistan revealed $40 \%$ overall ESBLs production rate. ${ }^{9}$

In 2005 a study in BSMMU, Bangladesh by Alim, showed that $23.19 \%$ of the Gram negative bacteria were ESBLs producing organism. ${ }^{10}$ While another study in the same institution in 2007 revealed ESBLs production rate to be $30.90 \%$ among the Gram negative bacteria. ${ }^{11} \mathrm{~A}$ similar study from Mymensingh, Bangladesh showed among the 300 gram negative isolates 214 (71.4\%) was ESBLs producers. ${ }^{12}$ These studies strongly suggest increasing prevalence of ESBLs producing organisms in Bangladesh.

So the present study was designed to see the rate the ESBLs production in gram negative bacteria by double disc synergy test and to analyze antimicrobial susceptibility of the ESBLs producing organisms.

\section{Materials and Methods}

\section{Settings and samples}

This study was carried out in the Microbiology \& Immunology Laboratory of Bangabandhu Sheikh Mujib Medical University (BSMMU), Dhaka during the period of July 2008 to June 2009. Total one hundred ten clinical Gram negative isolates were studied, which were isolated from different clinical samples (urine, wound swab, pus, throat swab and sputum) submitted to Microbiology \& Immunology Laboratory of BSMMU.

\section{Test for determination of ESBLs activity}

All 110 isolates were tested for ESBLs activity by Double Disc Synergy Test.

\section{Double Disc Synergy Test ${ }^{13}$}

Mueller Hinton agar plates were seeded with standardized inoculum of the test organism (corresponding to 0.5 McFarland tube). Amoxyclav (AMC, $20 \mu \mathrm{g}$ amoxicillin and $10 \mu \mathrm{g}$ clavulanic acid) disc was placed in the center of the inoculated plate. Three 3rd generation cephalosporin (ceftazidime CAZ 30 $\mu$ g,

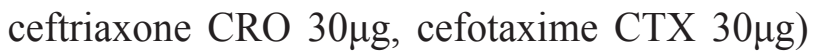
and one monobactam (aztreonam, AZT 30 $\mu \mathrm{g}$ ) discs were placed at $20 \mathrm{~mm}$ distance from amoxyclav disc. The plate was incubated overnight at $37^{\circ} \mathrm{C}$. Extension of the edge of the inhibition zone of ceftazidime, ceftriaxone, cefotaxime and aztreonam disc on the side exposed to the amoxyclav disc is positive for ESBLs production. This extension of edge of inhibition is due to synergy of disc of amoxyclav with the four discs used. (Fig. 1) 


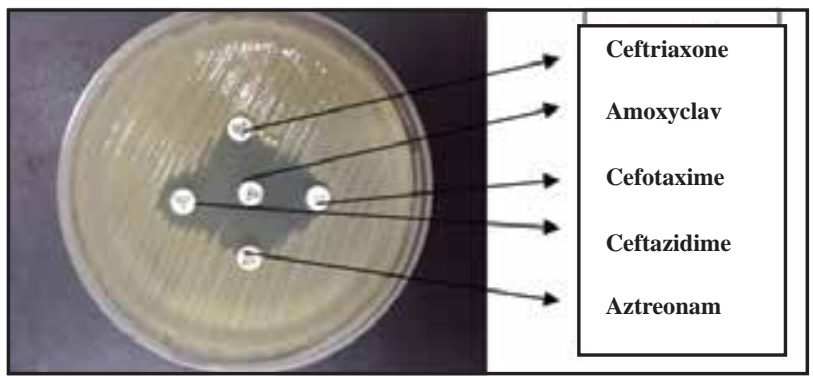

Fig. 1: Double disc synergy test positive for ESBLs production

\section{Antimicrobial sensitivity test: ${ }^{14}$}

All the ESBLs producing isolates were tested for antimicrobial sensitivity using disc diffusion technique by "Kirby-Bauer method" against Carbapenem and different non beta-lactam antimicrobial agents. ${ }^{11}$ These included Cotrimoxazole $\quad 1.25 / 23.75 \mu \mathrm{g} \quad$ (COT), Ciprofloxacin $5 \mu \mathrm{g}$ (CIP), Nitrofurantoin $300 \mu \mathrm{g}$

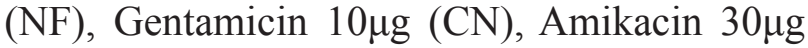

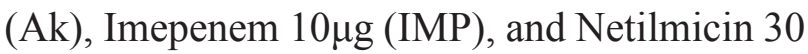
$\mu \mathrm{g}$ (NET). Susceptibility and resistance was determined based on the interpretative criteria recommended by the Clinical and Laboratory Standards Institute. ${ }^{12}$ E. coli ATCC 25922 was used as the quality control strain.

\section{Result}

In the present study, a total of 110 Gram negative isolates were isolated from various clinical samples of which majority were urine
60 (54.54\%), followed by wound swab $39(35.45 \%)$, pus $5(4.54 \%)$, throat swab $4(3.63 \%)$ and sputum 2 (1.18\%) (Fig. 2).

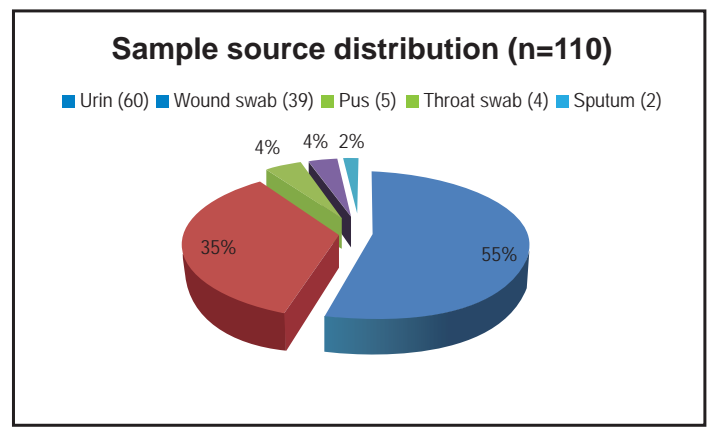

Fig. 2: Sample source distribution and percentage of total samples $(n=110)$

The distribution of different types of Gram negative bacteria among the total 110 isolates was as follows: Escherichia coli 61 (55.45\%), Klebsiella spp.14 (12.73\%), Pseudomonas spp 24 (21.81\%), Enterobacter 5 (4.54\%), Acinetobacter spp 4 (3.64\%) and Proteus spp 2 (1.82\%). All of these 110 Gram negative isolates were tested for ESBLs production by double disc synergy test and it was found that total 73 (66.36\%) isolates were ESBL producers. Among these ESBLs producers 49 (80.32\%) were Escherichia coli, 10 (71.42\%) Klebsiella spp., 6 (25\%) Pseudomonas aeruginosa, 4 (80\%) Enterobacter and 4 (100\%) Acinetobacter spp. (Table-1).

Table 1: Frequency of different isolates and their ESBLs production rate of total isolates $(n=110)$

\begin{tabular}{|l|c|c|}
\hline Organism & Number of isolates (\%) & ESBLs positive isolates (\%) \\
\hline E.coli & $61(55.45)$ & $49(80.32)$ \\
\hline Klebsiella spp. & $14(12.73)$ & $10(71.42)$ \\
\hline Pseudomonas spp. & $24(21.81)$ & $6(25)$ \\
\hline Enterobacter spp. & $5(4.54)$ & $4(80)$ \\
\hline Acinetobacter spp. & $4(3.64)$ & $4(100)$ \\
\hline Proteus spp. & $2(1.82)$ & $0(0)$ \\
\hline Total & $110(100)$ & $73(66.36)$ \\
\hline
\end{tabular}




\section{ORIGINAL ARTICLE}

Table 2: Rate of antimicrobial drug resistance among the ESBLs $(n=73)$

\begin{tabular}{|c|c|c|c|c|c|c|c|c|c|c|c|c|}
\hline \multirow{3}{*}{$\begin{array}{l}\text { Antimicrobial } \\
\text { agents } \\
\downarrow\end{array}$} & \multicolumn{12}{|c|}{ Bacterial Isolates } \\
\hline & \multicolumn{2}{|c|}{$\begin{array}{l}\text { Escherichia. } \\
\text { coli (49) }\end{array}$} & \multicolumn{2}{|c|}{$\begin{array}{l}\text { Klebsiella } \\
\text { spp (10) }\end{array}$} & \multicolumn{2}{|c|}{$\begin{array}{l}\text { Pseudomoas } \\
\text { spp (6) }\end{array}$} & \multicolumn{2}{|c|}{$\begin{array}{l}\text { Enterobacter } \\
\text { Spp (4) }\end{array}$} & \multicolumn{2}{|c|}{$\begin{array}{l}\text { Acinetobacter } \\
\text { Spp (4) }\end{array}$} & \multicolumn{2}{|c|}{$\begin{array}{l}\text { Total } \\
\text { ESBLs } \\
\text { producers }\end{array}$} \\
\hline & $\mathrm{n}$ & $\%$ & $\mathrm{n}$ & $\%$ & $\mathrm{n}$ & $\%$ & $\mathrm{n}$ & $\%$ & $\mathrm{n}$ & $\%$ & $\mathrm{n}$ & $\%$ \\
\hline Cotrimoxazole & 37 & 75.51 & 8 & 81.81 & 5 & 90.90 & 4 & 100 & 4 & 100 & 58 & 79.45 \\
\hline Ciprofloxacin & 48 & 97.95 & 8 & 81.81 & 4 & 81.81 & 4 & 100 & 4 & 100 & 68 & 93.15 \\
\hline Nitrofurantoin* & 7 & 14.28 & 2 & 25 & - & - & 3 & 75 & 3 & 75 & 15 & 25 \\
\hline Gentamicin & 33 & 67.37 & 9 & 90.90 & 5 & 90.90 & 4 & 100 & 4 & 100 & 55 & 75.34 \\
\hline Amikacin & 10 & 19.60 & 4 & 45.45 & 2 & 45.45 & 2 & 50 & 1 & 25 & 19 & 26.02 \\
\hline Imepenem & 0 & 0 & 0 & 0 & 0 & 0 & 0 & 0 & 0 & 0 & 0 & 0 \\
\hline Netilmicin & 16 & 32.65 & 4 & 45.45 & 1 & 27.27 & 4 & 100 & 2 & 50 & 27 & 36.98 \\
\hline
\end{tabular}

* Used for urine isolates only

Note: Figures in column 'n' represents the no. of resistant isolates in respective microorganism group.

The antimicrobial resistance pattern of the ESBLs producers showed an alarmingly high resistance to ciprofloxacin (93.15\%), cotrimoxazole (79.45\%), gentamicin (75.34\%) and netilmicin (36.98\%). Amikacin and, for urine isolates, nitrofurantoin showed fairly good sensitivity (26.02\% and 25\% resistance respectively). Imipenem was the only drug that was $100 \%$ sensitive ( $0 \%$ resistance) for all ESBLs producers. (Table 2)

\section{Discussion}

ESBLs producing organisms pose a major problem in clinical therapeutics. The incidence of ESBLs producing strains among clinical isolates has been steadily increasing over the past years, resulting in limitations of therapeutic options. ${ }^{15}$ The overall prevalence of ESBL producers was found to vary greatly in different geographical areas and in different institutes. 
In the present study $66.36 \%$ of the isolates were found to be ESBLs producer out of 110 Gram negative clinical isolates from different samples. But a similar study which was carried out 2 years earlier in 2007 in the same institute, BSMMU, by Rahman revealed ESBLs in only $30.90 \%$ strains of the Gram negative isolates. ${ }^{11}$ However a 2012 study from Mymensingh, Bangladesh detected 71.4\% ESBLs producers from 300 gram negative isolates. ${ }^{12}$ Higher (68\%) ESBLs production rate in enterobacteriaceae isolates has also been reported from India. ${ }^{16}$ One possible reason for such variation might be varying number of samples in different studies. ${ }^{17}$ The clinical condition of the source patients of samples might also be a contributing factor (acute versus chronic, past exposure to antimicrobials, etc.). The probable reasons of gradual increase in ESBLs detection in various Bangladesh studies might be 1) random and inappropriate use of $3^{\text {rd }}$ generation cephalosporin, which contribute to the evolution of ESBLs, ${ }^{3}$ 2) nonexistence of standard infection control practices in healthcare facilities and 3) Lack of national antibiotic policy.

Among the 110 isolates in the present study, 61 were E.coli, 24 Pseudomonas spp, 14 Klebsiella spp, 5 Enterobacter spp, 4 Acinetobacter spp and 2 Proteus spp and their rate of ESBLs positivity was 49 (80.32\%), 6 (25\%), 10 (71.42\%), 4 (80\%), 4 (100\%) and 0 (0\%) respectively. In 2007 Rahman from BSMMU, Dhaka, Bangladesh detected 35.38\% ESBL producers in E. coli, in Klebsiella spp 43.47\%, in Enterobacter spp $31.25 \%$, in Proteus spp $27.11 \%$, in Acinetobacter spp 26.32\% and in Pseudomonas spp $17.07 \%{ }^{11}$ Another study from Bangladesh (2010) showed 57.89\% ESBLs production for
Klebsiella spp. followed by Proteus spp. 50.0\%, E. coli $47.83 \%$ and Pseudomonas spp 31.35\%. ${ }^{18}$ A 2012 study from Mymensingh, Bangladesh showed that among 300 gram negative isolates 80\% Klebsiella spp $72 \%$ Proteus spp, $71 \%$ Enterobacter spp., $67.3 \%$ E. coli and $88.8 \%$ Pseudomonas spp were ESBL producers. ${ }^{12} \mathrm{~A}$ study from Tamil Nadu, India showed ESBLs producer in E. coli 77.35\%, Klebsiella spp 71.23\%, Pseudomonas spp 56.75\%, Salmonella spp 29.31\%, Enterobacter spp 35.48\%, and Proteus spp $34.61 \%{ }^{19}$ The reason of high rates of ESBLs production in all the strains in this study might be due to overall higher rate of ESBLs producer in the study isolates.

In the present study drug resistance of all ESBLs producer to most of the non beta-lactum antibiotics (cotrimoxazole, gentamicin, ciprofloxacin, amikacin) were found higher. This implies that ESBL producing organisms are multidrug resistant as genes that code for ESBLs are linked to other resistance genes. ${ }^{20}$ ESBLs producing isolates showed $100 \%$ sensitivity to imepenem which conforms clearly with the CDC (1999) ESBLs definition which says ESBLs are enzymes which hydrolyze $3^{\text {rd }}$ generation cephalosporins but sensitive to imipenem. ${ }^{21}$

\section{Conclusion}

In conclusion, screening for ESBLs production needs to be carried out routinely in every clinical diagnostic laboratory to guide clinicians in proper selection of antibiotics. Continued monitoring of the susceptibility pattern of ESBLs producing bacteria will provide invaluable information in clinical management of patients and, to control and prevent the spread of these type infections. 


\section{ORIGINAL ARTICLE}

\section{References}

1. Svard L. Evaluation of phenotypic and genotypic extended-spectrum beta-lactamase detection method. Uppsala Universitet, 2007:73-77.

2. Thomson KS. Controversies about Extended-spectrum and AmpC $\beta$-lactamase. Emerging Infectious Disease. 2001; 7(2):333-36.

3. Jacoby GA, Medeiros AA. More extended spectrum beta-lactamases. Antimicrobial Agents Chemotherapy. 1991;35:1697-1704.

4. Bradford PA. Extended-spectrum $\beta$-lactamases in the $21^{\text {st }}$ century: Characterization, epidemiology and detection of this important resistance threat. Clinical Microbiology Review. 2001;14(4):933-51.

5. Das A, Ray P, Garg. R, Kaur B. Extended-spectrum $\beta$-lactamase production in gram negative bacyerial isolates from cases of septicaemia. Silver Jubilee Conference. Conference book of All India Institute of Medical Sciences; New Delhi: 2001.

6. Pai H, Lyu S, Lee JH, Kim J, Kwon Y, Kim JW, et.al. Survey of extended-spectrum $\beta$-lactamases in clinical isolates of escherichia coli and klebsiella pneumoniae: prevalence of TEM-52 in Korea. Journal of Clinical Microbiology. 1999;37:1758-63.

7. Yan JJ, Wu SM, Tsai SH, Wu JJ, Su IJ. Prevalence of SHV-12 among clinical isolates of Klebsiella pneumoniae producing extended-spectrum $\beta$-lactamases and identification of a novel AmpC enzyme (CMY-8) in Souther Taiwan. Antimicrobial Agents and Chemotherapy. 2000;44:1438-1422.

8. Ho PL, Chow KH, Yuen KY, Ng WS, Chau PY. Comparison of novel, inhibitor potentiated disc diffusion test with other methods for the detection of ESBL in E.coli and K. pneumonia. Journal of Antimicrobial Chemotherapy. 1998;42:49 -54.

9. Jabeen K, Zafar A, Hasan R. Frequency and sensitivity pattern of extended spectrum beta lactamase producing isolates in a tertiary care hospital laboratory of Pakistan. JPMA. 2005;55:436.

10. Alim R. Thesis: detection of extended spectrum $\beta$-lactamases (ESBL) producing bacteria. Department of Microbiology and Immunology, BSMMU. 2005. 11. Rahman M. Rapid detection of extended spectrum $\beta$-lactamases production directly from primary culture. BSMMU. 2007.

12. Yasmin T. Thesis: Prevalence of ESBL among Esch. coli and Klebsiella spp. in a tertiary care hospital and molecular detection of important ESBL producing genes by multiplex PCR. Department of Microbiology, Mymensingh Medical College. 2012.

13. Performance Standards for Antimicrobial Susceptibility Testing; Sixteenth Informational Supplement, 2006. M100-S16, Clinical and Laboratory Standards Institute, Wayne, PA.

14. Baur AW, Kirby WMM, Sherris JC and Turck. Antibiotic susceptibility testing by a standardized single disc method. The American Journal of Clinical Pathology. 1966;36:493-96.

15. Ananthakrishnan AN, Kanungo R, Kumar A, Badrinath S. Detection of ESBL producers among surgical wound infections and burns patients in JIPMER Indian J Med Microbial. 2000;18(4):60-65.

16. Mathur P, Tatman A Das B, Dhawan B. Prevalence of extended beta lactamase producing gram negative bacteria in a tertiary care hospital. Indian J Med Res. 2002;120:553-6.

17. Agrawal P, Gosh AN, Satish K, Basu B, Kapila K. Prevalence of extended-spectrum $\beta$-lactamases among Escherichia coli and Klebsiella pneumoniae isolates in a tertiary care hospital. Indian J Pathol Microbiol. 2008;51:139-42.

18. Haque, R, Salam, MA. Detection of ESBL producing nosocomial gram negative bacteria from a tertiary care hospital in Bangladesh. Pk J Med Sci.

2010;26(4):887-91.

19. Vinodhini. Detection and Antimicrobial susceptibility pattern of ESBL producing gram negative bacteria. Asian J Pharm Clin Res. 2014;7 (Suppl 1):243-47.

20. Ahmed I, Salam A. Extended spectrum $\beta$-lactamases and bacterial resistance. Pakistan Journal of Medical Science. 200;18(2):151-55.

21. CDC. 1999. Laboratory Detection of Extended Spectrum beta-lactamases (ESBLs),

http://www.cdc.gov/ncidod/hip. 